\title{
具有特殊甾核结构甾体化合物的合成及生理活性研究进展
}

\author{
甘春芳盛海兵戚斌斌杨雷 \\ 赵丹丹黄燕敏熣建国* \\ (广西师范学院化学与生命科学学院 南宁 530001)
}

\begin{abstract}
摘要 改变甾体的甾核结构，在甾核或支链中引入不同的官能团，可以得到一些具有不同生理活性的化合物. 按照不 同类型甾核结构分类，结合本课题组在具有特殊甾核结构甾体化合物的合成和生理活性研究方面所取得的一些成果， 对近年来具有 A-失碳甾体化合物、B-失碳甾体化合物及 C-失碳-D-增碳甾体化合物的合成及生理活性进行讨论，并展 望了此类化合物的发展趋势及应用前景.
\end{abstract}

关键词＼cjkstart特殊甾核; A-失碳甾体化合物; B-失碳甾体化合物; C-失碳-D-增碳甾体化合物

\section{Recent Advance of Synthesis and Bioactivities for Steroids with Special Steroidal Nucleus}

\author{
Gan, Chunfang \\ Sheng, Haibing \\ Qi, Binbin \\ Yang, Lei \\ Zhao, Dandan \\ Huang, Yanmin \\ Cui, Jianguo* \\ (College of Chemistry and Life Science, Guangxi Teachers Education University, Nanning 530001)
}

\begin{abstract}
By changing the structure of steroidal nucleus, or introducing different functional groups into steroids, some steroidal compounds with various bioactivities might be obtained. In this paper, according to the different types of steroid nucleus structure, combining with the results of our research in steroids with special steroidal nucleus, the advance of synthesis and biological activities for A-nor steroids, B-nor steroids and C-nor-D-homo-steroids is discussed in recent years, and the development trend and application prospect are forecasted.
\end{abstract}

Keywords special steroidal nucleus; A-nor steroids; B-nor steroids; C-nor-D-homo-steroids

甾体化合物是一类对生命体具有重要生理功能的 化合物，也被称为生命的荷尔蒙. 它们具有环戌烷并全 氢化菲的结构, 该结构俗称甾体化合物的甾核. 在甾核 的10-位和 13-位，分别具有一个甲基取代，称为角甲基， 同时在甾核的 17 -位具有一根支链. 甾核是甾体化合物 的特征结构, 甾核结构的改变, 会对甾体化合物的生理 功能产生较大变化. 如对甾核中的环进行扩环或缩环, 或改变甾体化合物甾核中环的结构、或在甾核中引入不 同的杂原子、取代基及改变其支链结构, 都会对其生物 活性产生重要影响.

本文主要对近年来一些具有特殊的增碳或失碳环 甾核结构甾体化合物的合成及生理活性进行综述.

\section{A-环失碳甾体化合物}

A-环失碳甾体化合物对啮齿类动物具有明显的抗 着床作用, 并且此作用和雌激素活性有一定相关性. 由 于我国实行计划生育政策, 因此我国研究人员从 20 世 纪 60 年代起就对此类化合物进行了大量的化学合成与 药理学研究 ${ }^{1]}$, 认为其有发展成为探亲避孕药或紧急事 后避孕药的潜力, 最终优选了 $\mathrm{A}$-失碳甾体丙酸酯衍生 物 $2 \beta, 17 \beta$-二羟基- $2 \alpha, 17 \alpha$-二乙炔基-A-失碳- $5 \alpha$-雄甾烷$2 \beta, 17 \beta$-二丙酸酯 $[1$, Anordrin, Chart 1, 双炔失碳酯, 抗 孕(探亲) 53 号]作为新型探亲避孕药进入临床研究.

\footnotetext{
*E-mail: cuijg1954@126.com

Received March 18, 2014; revised April 21, 2014; published online May 7, 2014.

Project supported by the Natural Science Foundation of Guangxi Province (Nos. 2012GXNSFAA053018, 2012GXNSFAA053018) and the Scientific and Technology Research Funding of Guangxi University (No. 200103YB067).

广西省自然科学基金(Nos. 2012GXNSFAA053018, 2012GXNSFAA053018)及广西高等科学技术研究(No. 200103YB067)资助项目.
} 


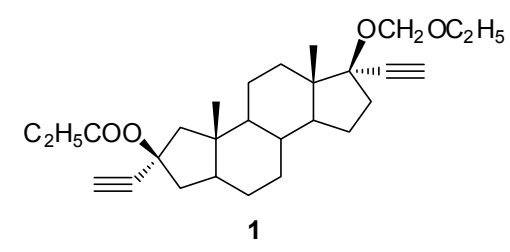

Chart 1

但是, 双炔失碳酯作为紧急避孕药效率不高, 生物 利用度较差(仅为 $1 \%$ ), 临床用量较大, 有较明显的雌激 素样副作用. 双炔失碳酯除了对多种动物及人具有显著 的抗生育功能外, 还具有一定的抗肿瘤和抗新生血管生 成作用 ${ }^{[2]}$.

刘静等 ${ }^{[3]}$ 以双酮 2 (Chart 2)为原料, 结合双烯孕腈 的药物结构特点对双炔失碳酯进行结构改造, 合成了 6 个新型 A-失碳甾体化合物, 并进行体外生物试验, 发现 3a 和 3b (Chart 2)两个化合物的体外生物活性高于双炔 失碳酯.

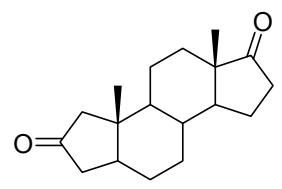

2

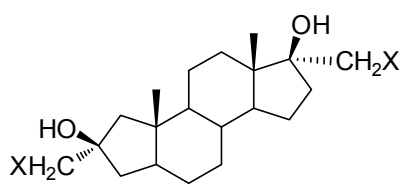

3

3a: $X=N_{3}, 3 b: X=C l, 3 c: X=B r$<smiles>Cc1cccc(O[14c]2ccc(O)cc2)c1</smiles><smiles>Cc1ccc(O)cc1C</smiles>

\section{Chart 2}

张培翼等 ${ }^{[4]}$ 以化合物 $\mathrm{A}$-失碳-17-羰基-5 $\alpha$-雄甾- $2 \alpha$ 乙炔基- $2 \beta$-羊基(4)为起始原料, 通过对 4 进行结构改造, 得到 8 个含 $\mathrm{N}$ 或 $\mathrm{S}$ 的 $\alpha$-双炔失碳醇类似物 $\mathbf{6 a} \sim \mathbf{6 h}$ (Scheme 1), 并对合成的化合物进行体外黄体细胞抑制 活性试验, 结果表明, 其中化合物 6c, 6d, 6f 的体外抑制 黄体细胞活性高于双炔失碳醇. 因此, 从具有此类结构 特征的化合物中获得比双炔失碳醇活性更高、副作用更 小的甾体避孕药, 值得进一步深入研究.

氧甲氢龙(oxandrolone)商品名为 Anavar, 是由美国 Searle 实验室研发, 并于 1964 年在美国上市的一种促蛋 白同化甾体激素, 它对人体碳水化合物诱导的甘油三酯 过多具有显著疗效 ${ }^{[5]}$, 其作用机制为抑制乙酸转化为甲 基二羟戊酸, 从而减少胆固醇的生成. 主要用于降血 脂、术后和严重烧伤的肌肉恢复、艾滋病、Turner 综合 症以及辅助用于骨质疏松症的治疗. 该化合物结构中含 有一个A-环内酯结构, 可通过 A-失碳五环酮的 Baeyer-

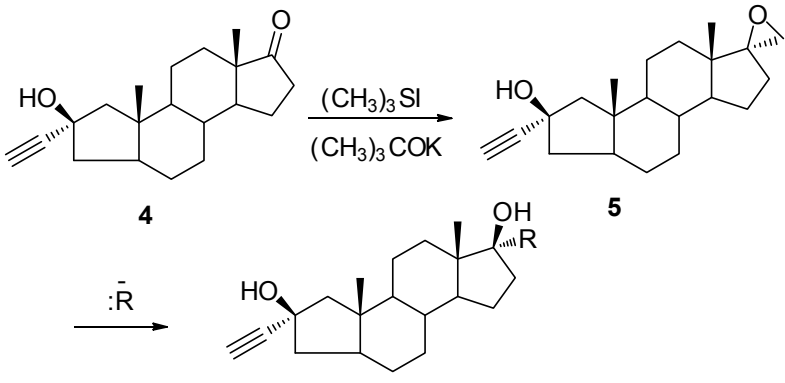

6

$6 \mathbf{a}: \mathrm{R}=\mathrm{C} \equiv \mathrm{CH}, \mathbf{6 b}: \mathrm{R}=\mathrm{H}_{2} \mathrm{C}-\mathrm{N}, \mathbf{2} \mathbf{c}: \mathrm{R}=\mathrm{H}_{2} \mathrm{C}$

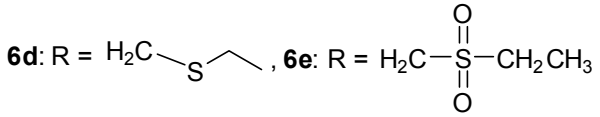<smiles>CCSc1ccc([As][R3]=O)cc1</smiles><smiles>CS(=O)(=O)c1ccc(F)cc1</smiles>

\section{Scheme 1}

Villiger 反应得到. 李宗桃等 ${ }^{[6]}$ 以表雄酮为原料, 经 8 步 反应合成了目标物氧甲氢龙，总收率达 $21 \%$ (Scheme 2).

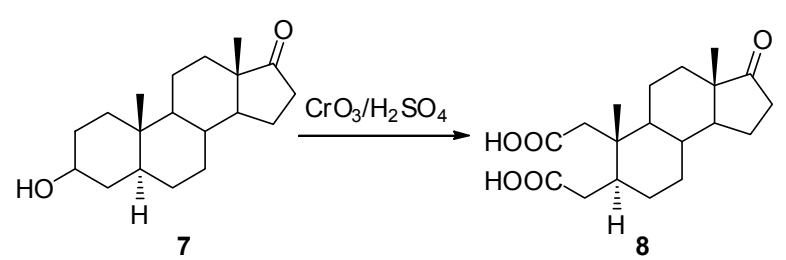

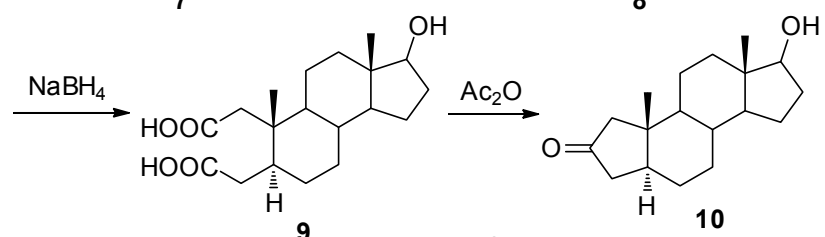

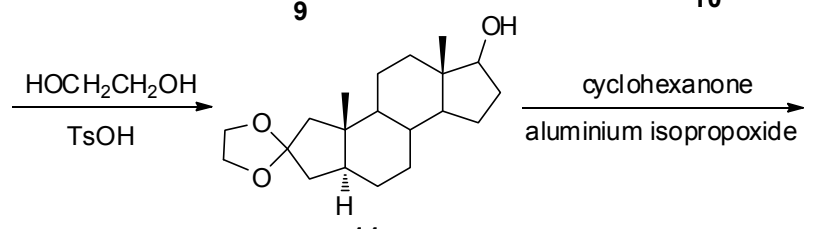

11

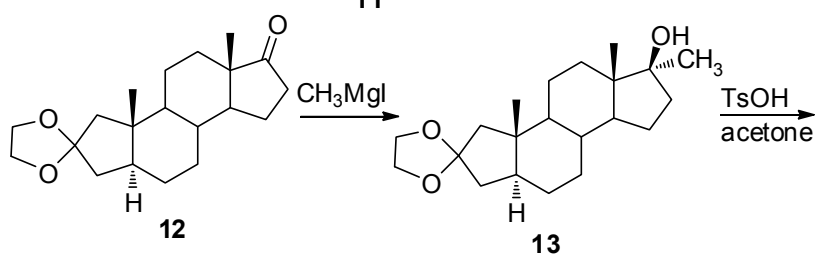<smiles>C[C@H]1CCC2C(CC[C@@]3(C)C2CC[C@@]3(C)O)C1CC(=O)O</smiles>

Scheme 2 
另外, 他们 ${ }^{[7]}$ 进一步以化合物 $\mathbf{1 0}$ 为原料, 通过与不 同的酰氯反应，得到化合物 10 的某些 17-酯基衍生物, 此中间体进一步经 Baeyer-Villiger 反应得到氧甲氢龙的 系列衍生物 17a $\sim 17 \mathbf{h}$ (Scheme 3).

分子化石(化石生物标记)是揭示现代有机地球化学 和海洋开发的重要工具. 它们的结构、分布模式和反应 途径能够揭示地质历史. 在最近研究中, 质谱数据表明 早古生代原油和沉积岩盐中存在 3 个明显分离的异构体 (气体色谱法), 推测为碳骨架修饰的甾烷化合物和常规 甾萜类化合物及重排甾烷类 $\mathrm{C}_{19}$ 和 $\mathrm{C}_{20}$ 甾体化合物 ${ }^{[8]}$, 这 些化合物有可能是化石生物标志物 ${ }^{[9]}$, 其精确结构能够 追溯到潜在的前体生物. Norden 等 ${ }^{[10]}$ 设计合成了化合物 22 (Scheme 4), 并将其与未知物的质谱进行对照, 化合 物 22 是一个 A-失碳甾体化合物.
在 Scheme 4 中, 用 $\mathrm{CrO}_{3}$ 氧化断裂 3-酮 $\mathbf{2 0}$, 然后通 过 Dieckmann 缩合或者 Favorskii 重排可以得到 A-失碳 甾烷. 作者采用三水铊三硝酸酯(TTN)进行缩环反应, 化合物 20 在 $\mathrm{CH}_{2} \mathrm{Cl}_{2}$ 中与过量的 TTN 在室温下反应，几 小时就可以将原料完全转化为产物 21. 21 进一步通过脱 羧得到 A-失碳甾烷 22.

Nowrouzi 等 ${ }^{[11]}$ 通过 6 步反应合成了另一个 A-失碳 甾烷 28 (Scheme 5), 合成总产率为 $15 \%$. 化合物 $\mathbf{2 8}$ 与 22 相比, 28 不存在 17-甲基, 而是在 A-环的 2-位上存在 一个甲基，它们都属于 $\mathrm{A}$-失碳甾烷.

为了与从阿曼 $(O m a n)$ 得到的地质未知化石中的 $\mathrm{C}_{19} \mathrm{H}_{32}$ 有机生物标志物相比较, 该课题组设计合成了另 一个 A-失碳雄甾烷异构体 35 (Scheme 6).

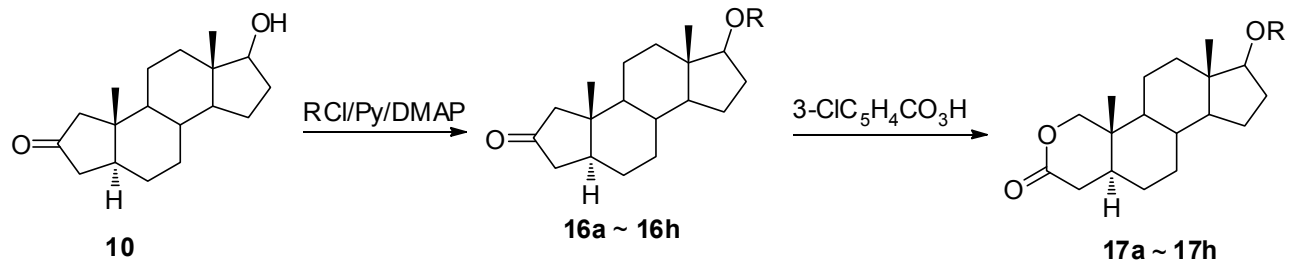<smiles>CC(=O)COc1ccc([13C](=O)COc2ccc(Br)cc2)cc1</smiles>

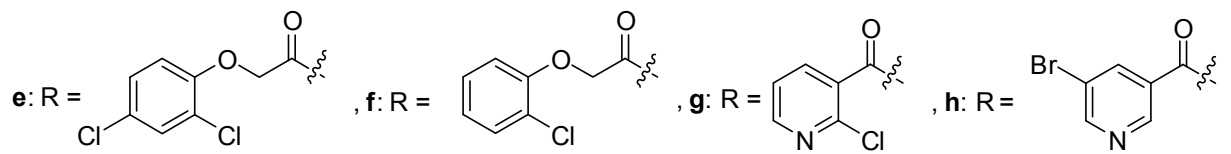

Scheme 3

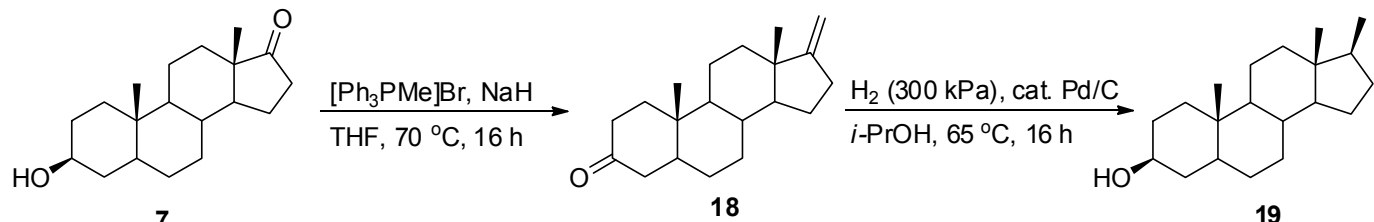

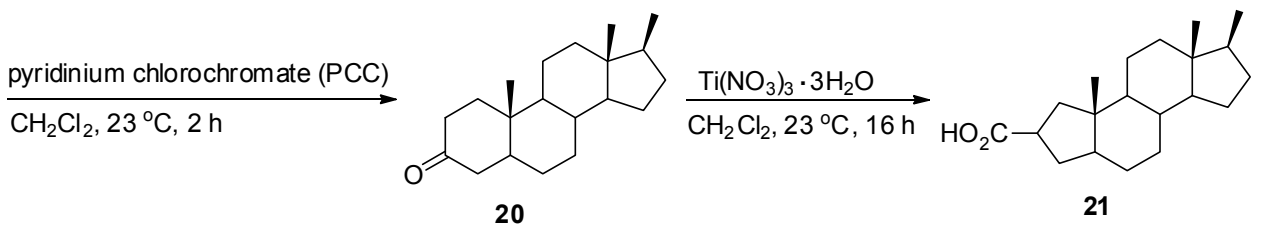

(1) dicyclohexylcarbodiimide (DCC), cat. 4-(dimethylamino)pyridine (DMAP) $\mathrm{CH}_{2} \mathrm{Cl}_{2}, 23^{\circ} \mathrm{C}, 5 \mathrm{~min}$

(2) 1-hydroxy-2-thiopyridone, $23^{\circ} \mathrm{C}, 2.5 \mathrm{~h}$ (exclusion of light)

(3) $\mathrm{Bu}_{3} \mathrm{SnH}$, cat. 2,2-azobis(isobutyronitrile) (AlBN), $\mathrm{C}_{6} \mathrm{H}_{6}, 85^{\circ} \mathrm{C}, 16 \mathrm{~h}$

(4) $\mathrm{KOH}, \mathrm{H}_{2} \mathrm{O}, 23^{\circ} \mathrm{C}, 0.5 \mathrm{~h}$

(5) $\mathrm{HCl}, \mathrm{H}_{2} \mathrm{O}, 23^{\circ} \mathrm{C}, 2 \mathrm{~h}$

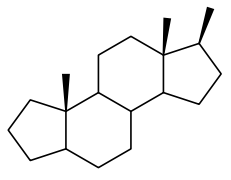

22

\section{Scheme 4}



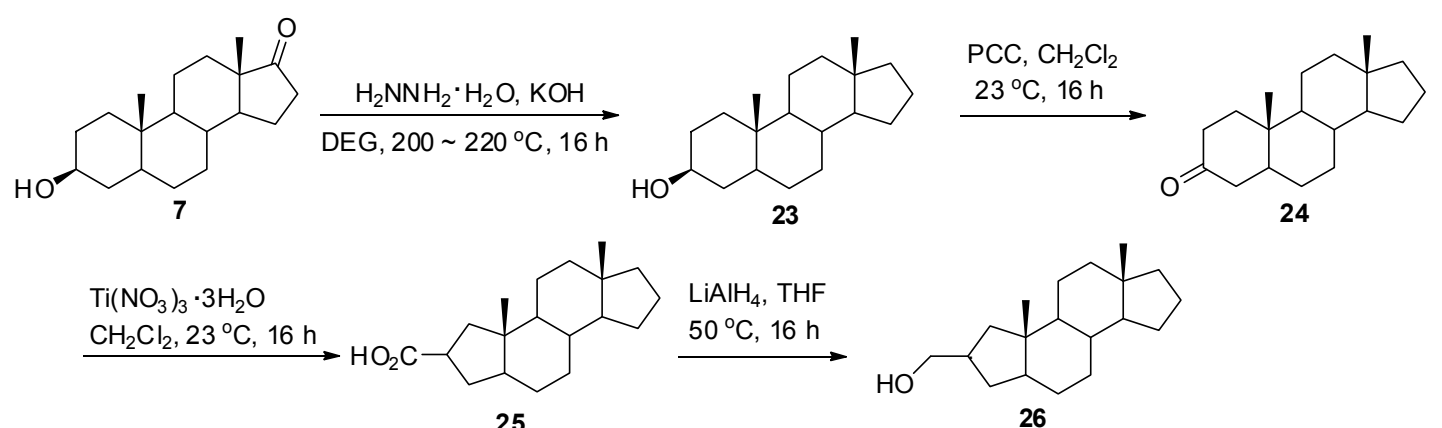

25

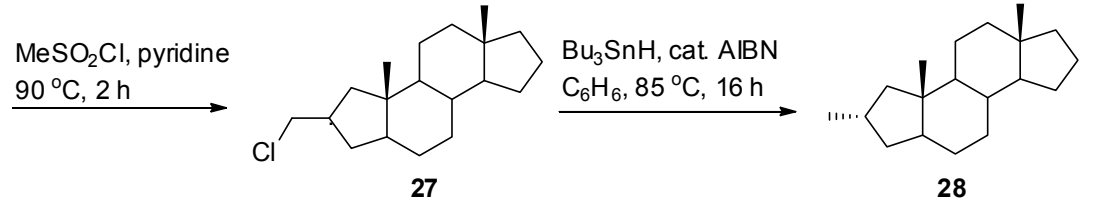

Scheme 5
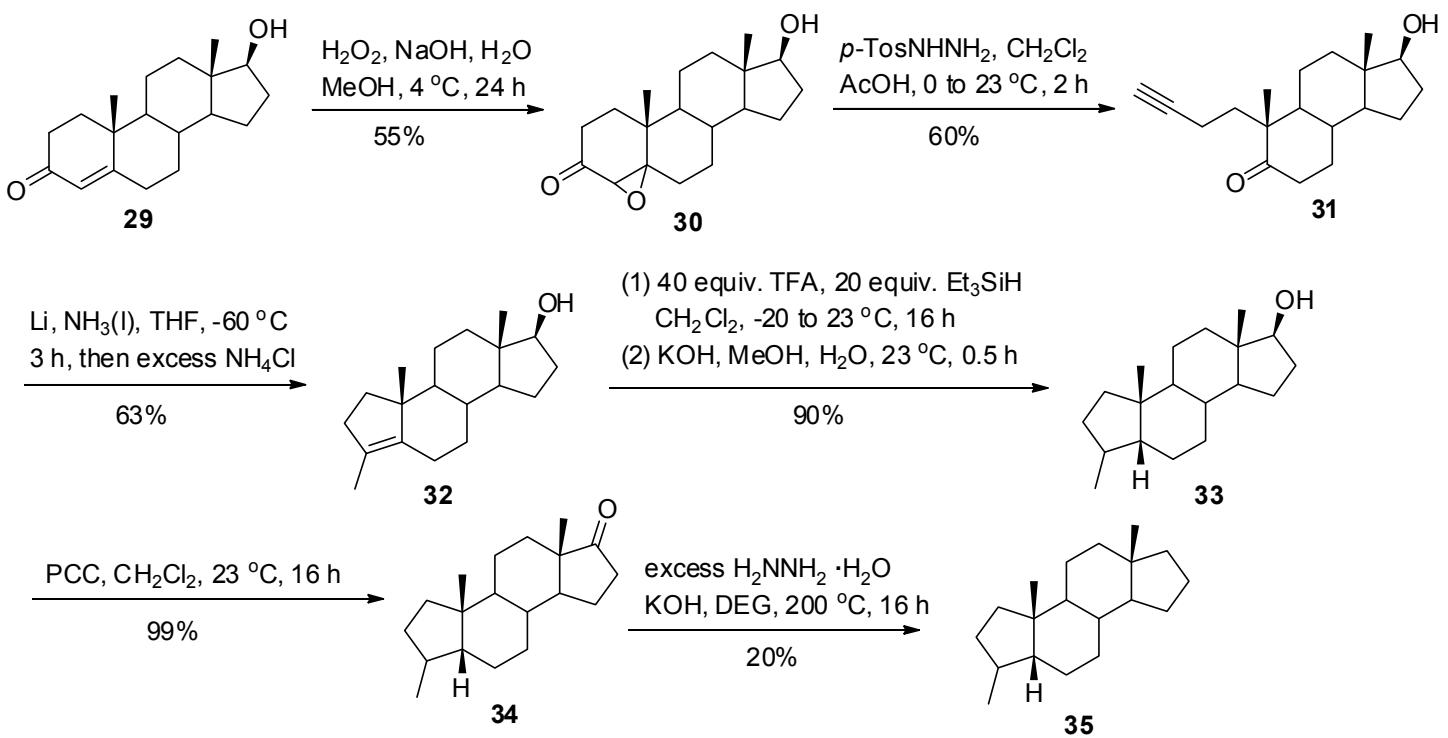

dr $27 / 8 / 53 / 12$

Scheme 6

在天然产物中也存在 A-失碳甾体化合物, Qiu 等 ${ }^{[2]}$ 在中国海南岛采集的海绵 Acanthella cavernosa 中分离 得到 $\mathrm{A}$-失碳甾体化合物 36 38 (Chart 3), 这 3 个化合物 对藤壶 Balanus albicostatus 显示出一定的抗污活性, 其 $\mathrm{EC}_{50}$ 值分别为 $8.2,23.5,31.6 \mu \mathrm{g} / \mathrm{mL}$.
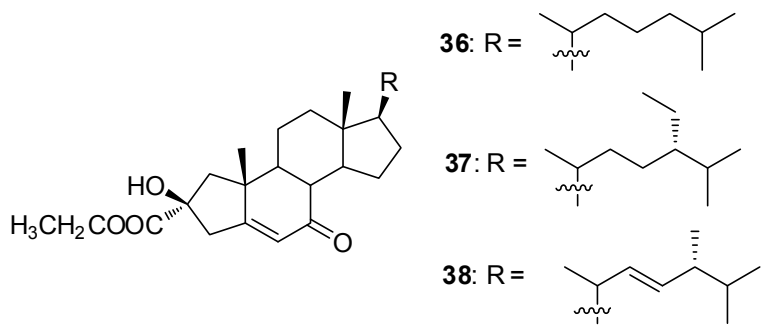

Chart 3

另外, Pointinger 等 ${ }^{[13]}$ 从 6 种不同来源的米兰 Aglaia silvestris 根、茎、叶中提取分离得到另外一些 $\mathrm{A}$-失碳甾 体化合物 39, 40 (Chart 4). 由此可见, A-失碳甾体化合 物也存在于自然界中, 它们的生成可能是相应的普通甾 体化合物发生降解所形成.

\section{B-环失碳甾体化合物}

在自然界中也存在 B-环失碳甾体化合物. Higuchi 研究小组 ${ }^{[14]}$ 在日本海绵 Stelletta hiwasaensis 中分离得到 化合物 41 (orostanal, Chart 5), 在 $10 \mu \mathrm{g} / \mathrm{mL}$ 浓度下，化 合物 41 可以诱导 HL-60 细胞调亡, 并且在 $1.7 \mu \mathrm{mol} \cdot$ $\mathrm{L}^{-1}$ 可以抑制 $50 \%$ 的细胞生长. 周维善等 ${ }^{[15,16]}$ 从相对易 得的原料猪去氧胆酸出发, 经过 12 步反应, 首次合成得 到总产率为 $18 \%$ 的化合物 $\mathbf{4 1}$, 产物结构与天然分离产 物结构相一致. 


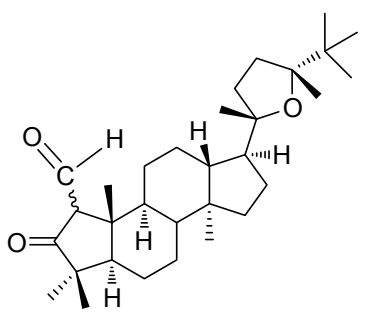

39 silvagin $\mathrm{A}(1 \mathrm{H}-\alpha)$ $20 S, 24 R$

39a silvagin $B$

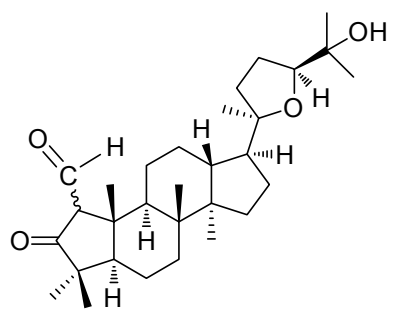

40 Isosill vagin $\mathrm{A}(1 \mathrm{H}-\alpha)$ 20R,24R

$40 \mathrm{a}$ isosill vagin $\mathrm{B}(1 \mathrm{H}-\beta)$

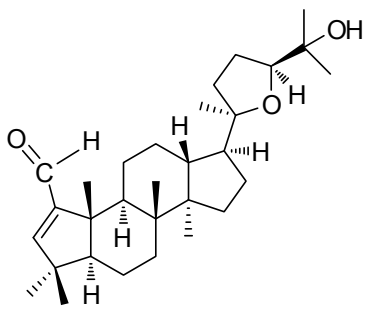

40b desoxysilvagin

\section{Chart 4}

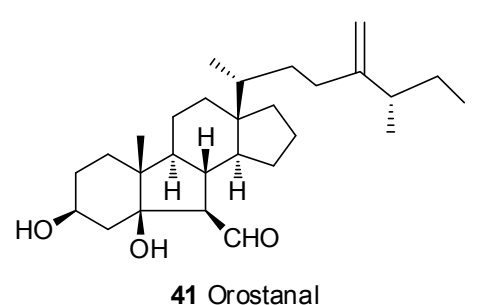

Chart 5

从结构上看, 具有 [6-5-6-5]-稠环结构类型的 B-环 失碳甾体化合物在自然界中比较罕见, 这类化合物多从 甾体化合物的臭氧化反应中得到. 胆固醇的臭氧化产物 有可能作为 $\mathrm{O}_{3}$ 的生物标志, 所形成产物可通过与 2,4二硝基苯肼(DPNH)的反应进行捕获跟踪. Wentworth 等 ${ }^{[17]}$ 研究表明, 在人体的某些疾病中, 由于臭氧的产生 导致胆固醇的氧化，最终会形成具有 [6-5-6-5]稠环结构 的 $\mathrm{B}$-失碳甾体衍生物 3 $3,5 \beta$-二羟基- $6 \beta$-甲酰基-B-降胆 甾烷(42) (Chart 6). 他们在其专利中 ${ }^{[18]}$ 报道了一系列具 有[6-5-6-5]-甾核结构的 B-失碳胆甾烷衍生物 $\mathbf{4 2} \sim \mathbf{5 1}$, 使用这些胆固醇的 B-失碳甾体衍生物作为识别剂, 可 用于诊断动脉䋆样硬化和其他炎症性疾病.

Suñol 等 ${ }^{[20]}$ 合成了系列孕甾醇酮的 B-失碳衍生物 54 64 (Scheme 7), 同时对合成物进行了 $\gamma$-氨基丁酸 $\mathrm{A}$ $\left(\mathrm{GABA}_{\mathrm{A}}\right)$ 受体研究. 为增加产物的水溶性, 在分子中引 入了环氧原子或羰基. 合成路线见 Scheme 7, 通过 Mitsunobu 反应, $3 \beta$-羊基转变为 $3 \alpha$-羟基.

Wei 等 ${ }^{[21]}$ 在分离提取加勒比海海绵 Svenzea zeai 中 的活性物质时分离得到 $0.007 \%$ 的化合物 64 和 $2.2 \%$ 的化

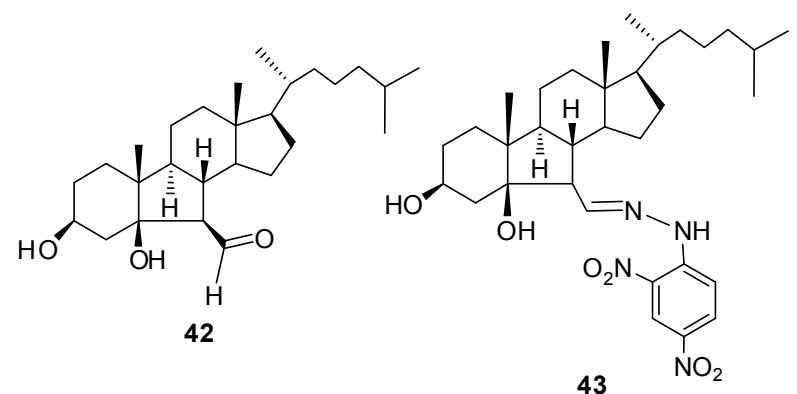

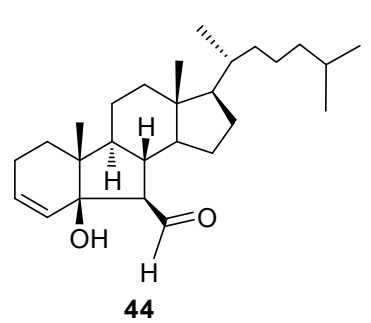

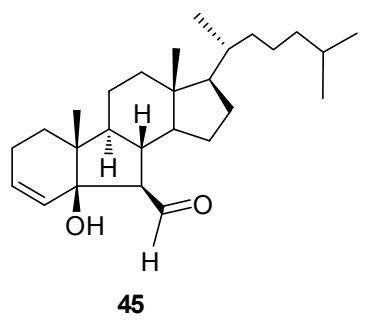

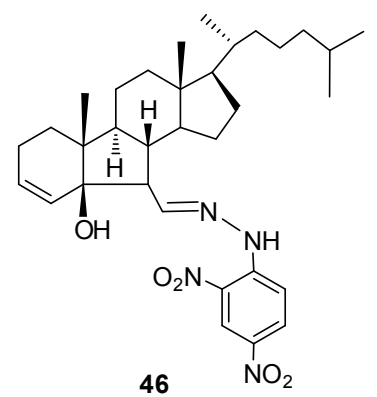<smiles>[Z17]C(=O)C1=C2C=CCC[C@]2(C)[C@H]2CC[C@]3(C)C(CC[C@@H]3[C@H](C)CCCC(C)C)[C@H]12</smiles>

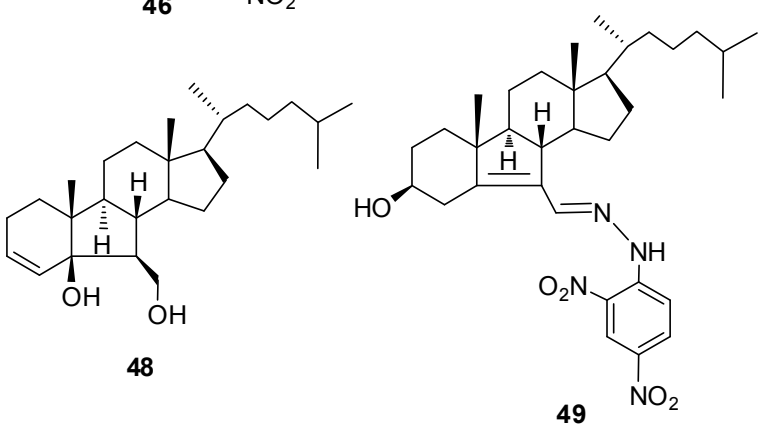

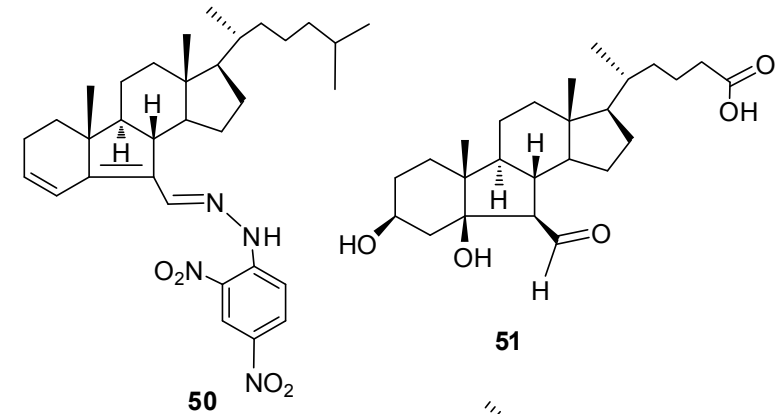

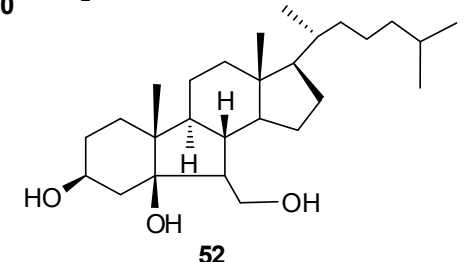

Chart 6 


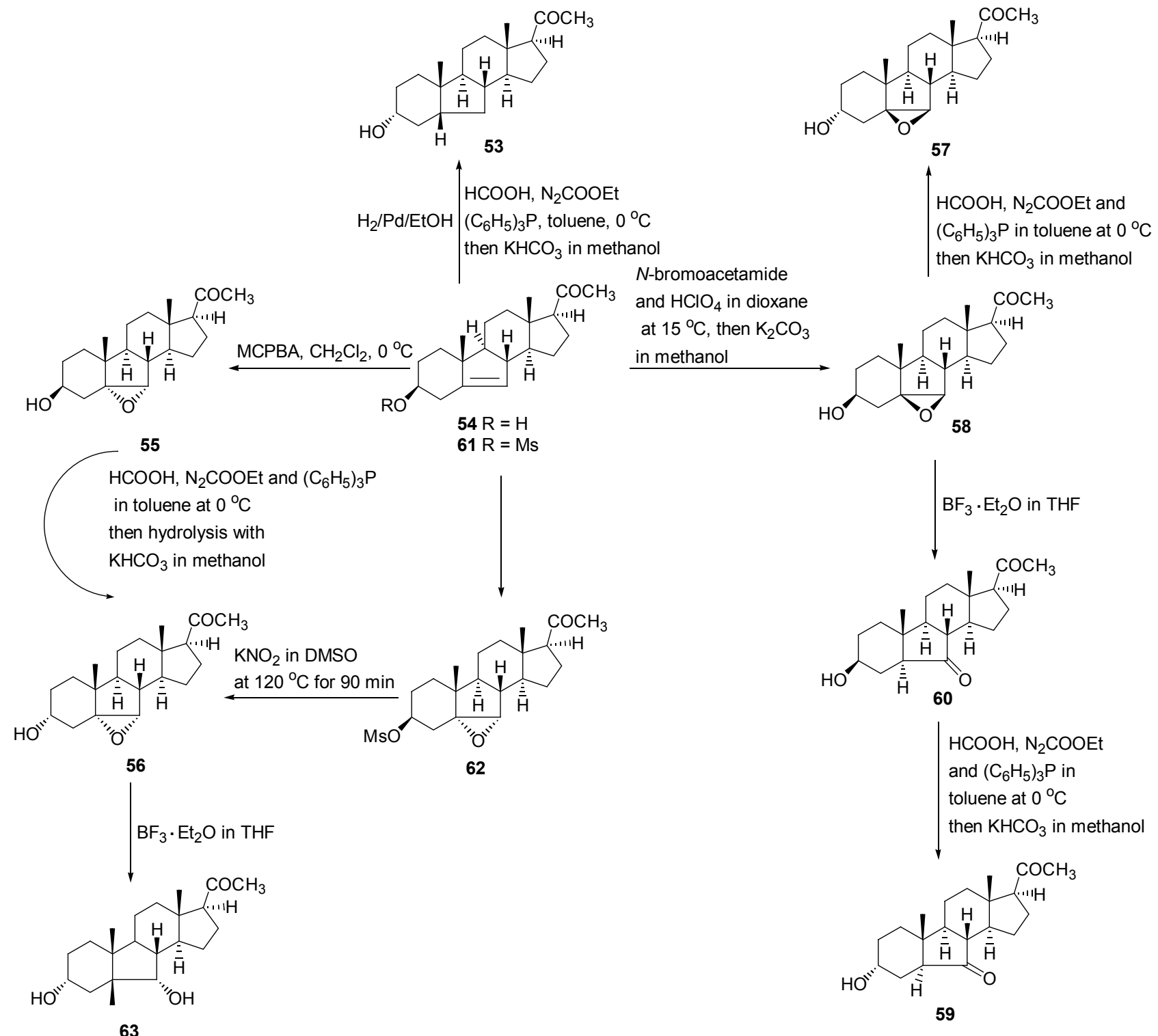

Scheme 7

合物 65 (Chart 7), 在进行生理活性实验中发现, 化合物 65 对抗结核分支杆菌最低抑菌浓度(MIC) 值为 120.1 $\mu \mathrm{g} / \mathrm{mL}, 64$ 为 $7.8 \mu \mathrm{g} / \mathrm{mL}$. 该课题组进一步合成了系列具 有[6-5-6-5]-甾核结构的甾体化合物, 并将它们与 B 环为 六元环结构的甾体化合物进行比较, 测定了它们抗结核 分支杆菌的 MIC 值, 另外还试验了化合物 64 74 的体 外细胞毒活性 $\left(\mathrm{IC}_{50}\right)$. 研究结果表明, 具有六元 B 环结构 甾体化合物 $(65,67,69,71,73)$ 缺乏明显的抑制肺结核分 支杆菌活性 $\left(\mathrm{IC}_{50}>128 \mu \mathrm{g} / \mathrm{mL}\right)$. 然而, 具有五元 B 环结 构的类似物 $(64,66,68,70,72)$ 显示明显的活性 $\left(\mathrm{IC}_{50}\right.$ 值: $26.6 \sim 54.7 \mu \mathrm{g} / \mathrm{mL}$ ). 同时, 具有[6-5-6-5]-结构的 B-环失 碳甾体化合物也显示出一定的细胞毒性, 而 B 环为六元 环的化合物基本无活性.
Shukla 等 ${ }^{[22]}$ 从植物 Hoodia gordonii 中提取分离出 Hoodistanal (75)和 Dehydrohoodistanal (76) (Chart 8)两种 具有[6-5-6-5]-稠环结构的甾体化合物，这两者都存在一 个 20-差基取代基. Cygan 等 ${ }^{[23]}$ 探讨了具有[6-5-6-5]-甾 核结构的甾体化合物 75 和 76 与蛋白质作用的机理及其 生物活性.

我们课题组 ${ }^{[24-27]}$ 对具有 [6-5-6-5]-甾核结构的 B-环 失碳甾体化合物进行研究, 设计合成了系列具有不同结 构特征的 B-环失碳甾体化合物，同时对其体外抗肿瘤 活性进行了评估. 从简单易得的原料胆甾醇、谷甾醇、 去氢表雄酮和孕烯醇酮出发, 采用 Scheme 8 中的合成 路线, 合成得到 B-失碳甾体化合物 81 86. 


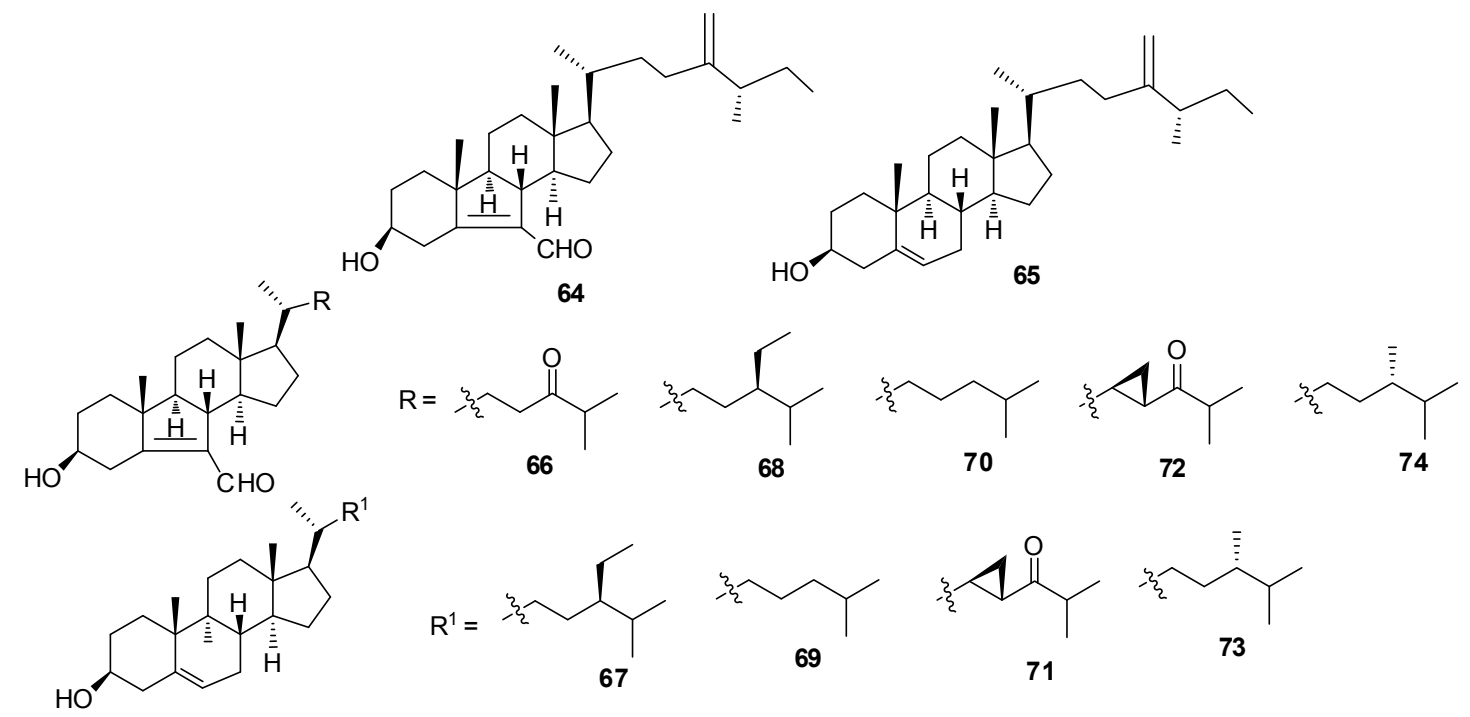

Chart 7
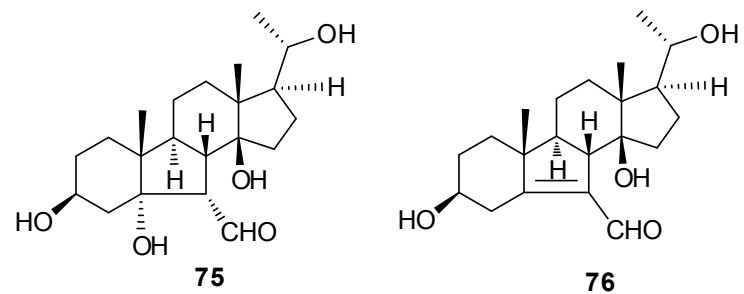

Chart 8

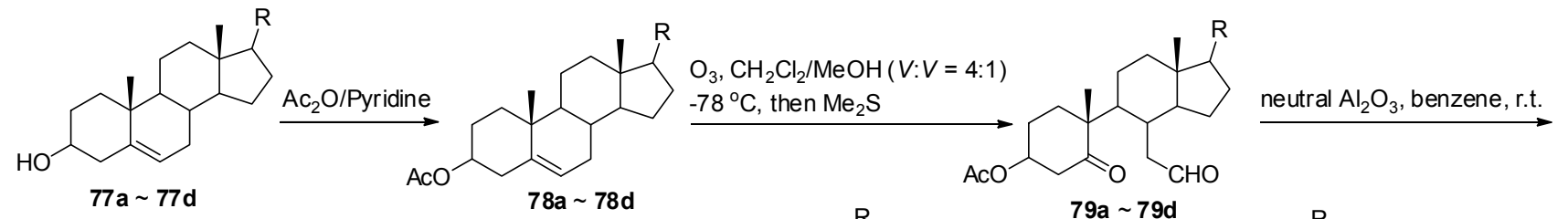

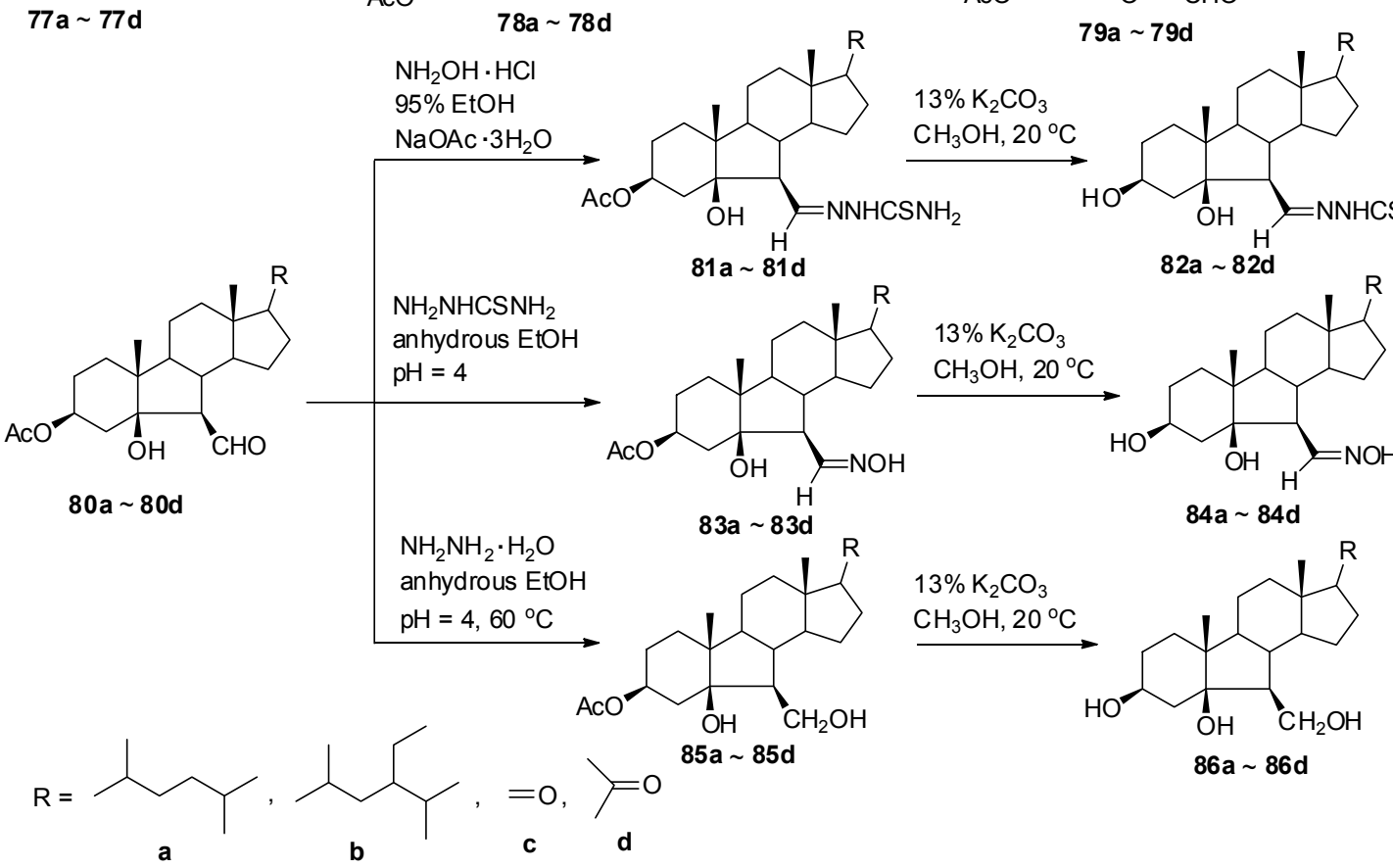

Scheme 8 
在上述反应中, 在室温下脱去乙酰基时, 得到 5-羟 基保留的产物 82,84 和 86 . 但是, 当提高反应温度, 在 $45{ }^{\circ} \mathrm{C}$ 对化合物 80a 水解脱去 3-乙酰基时, 同时发生了 5-羟基的脱水反应，生成 $\alpha, \beta$-不饱和羰基化合物 87, 87 进一步通过 6-醛基的官能团转换得到化合物 $88 \mathrm{a} \sim \mathbf{8 8 c}$; 进一步提高水解温度, 在 $60{ }^{\circ} \mathrm{C}$ 对 $80 \mathrm{a}$ 进行水解时, 主 要得到 3-乙酰氧基和 5-差基同时消除的产物 89, 化合物 89 的 6-醛基进一步通过官能团转换得到化合物 90a 99c (Scheme 9).

采用 MTT 法, 分别使用人卵巢癌 $(H e L a)$ 、肝癌 (Bel-7404)和胃癌(SGC-7901)细胞株对合成物进行抑制 肿瘤细胞生长增殖活性试验, 结果见表 1 所示. 从表 1 结果可知, 具有胆甾烷和谷甾烷支链结构的此类化合物 对这三种肿瘤细胞都显示出明显的细胞毒性, 可以抑制 肿瘤细胞的生长增殖. 而另外两种类型支链的此类化合 物对这些细胞没有表现出明显的生长抑制作用. 其中, 具有胆甾支链结构、6-位为缩氨硫腙基时的化合物 81a
及 88a 抑制活性最好, 81a 对 HeLa, Bel-7404, SGC-7901 肿瘤细胞的 $\mathrm{IC}_{50}$ 值分别为 $19,8.6,8.4 \mu \mathrm{mol} / \mathrm{L}$.

表 1 某些 B-失碳甾体化合物的体外抑制肿瘤细胞生长增殖 活性 $\left(\mathrm{IC}_{50}, \mu \mathrm{mol} / \mathrm{L}\right)$

Table 1 Antiproliferative activity of some B-nor steroids $\left(\mathrm{IC}_{50}\right.$, $\mu \mathrm{mol} / \mathrm{L})$

\begin{tabular}{cccc}
\hline \multirow{2}{*}{ 化合物 } & \multicolumn{3}{c}{ 肿瘤细胞株 } \\
\cline { 2 - 4 } & HeLa & Bel-7404 & SGC-7901 \\
\hline $\mathbf{8 0 a}$ & 57 & 40 & 36 \\
$\mathbf{8 1 a}$ & 19 & 8.6 & 8.4 \\
$\mathbf{8 5 a}$ & 61.3 & 25 & 19.9 \\
$\mathbf{8 8 a}$ & 13 & 11 & 9.0 \\
$\mathbf{9 0 a}$ & $>200$ & $>200$ & $>200$ \\
$\mathbf{8 0 b}$ & 56 & 12 & 33 \\
$\mathbf{8 0 c}$ & $>200$ & $>200$ & $>200$ \\
$\mathbf{8 0 d}$ & 119 & $>200$ & 146 \\
$\mathbf{8 1 b}$ & 13 & 11 & 10 \\
$\mathbf{8 1 c}$ & $>200$ & $>200$ & $>200$ \\
$\mathbf{8 1 d}$ & $>200$ & $>200$ & $>200$ \\
\hline
\end{tabular}

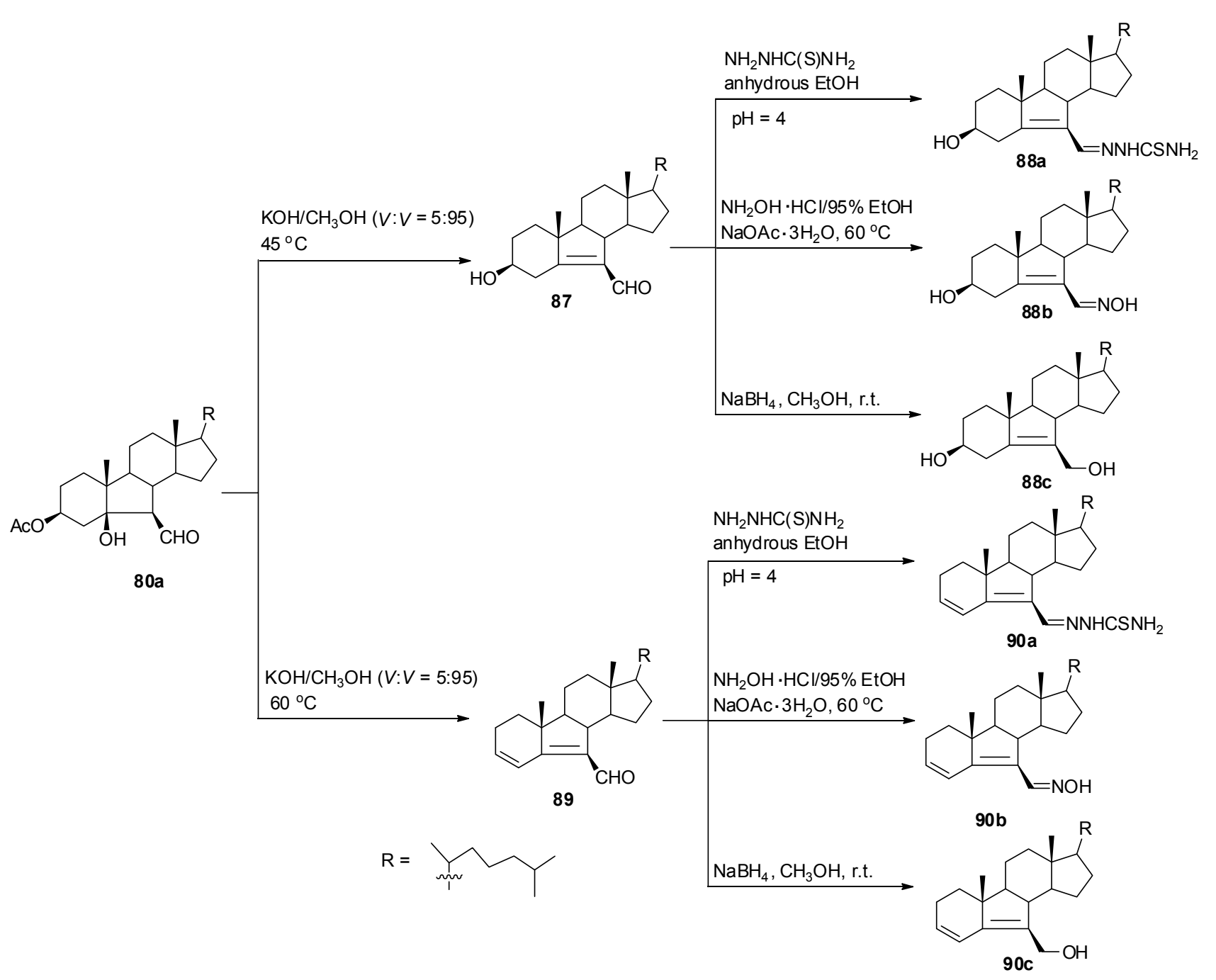

Scheme 9 
由于具有胆甾支链结构的此类甾体化合物细胞毒 性较好，我们进一步设计合成了一些具有烷基或苯基取 代氨基硫腙基的 B-失碳胆甾烷类化合物 $91 \sim 98^{[28]}$, 并 合成了茮氧肜醚化合物 99, 100 (Scheme 10)和 6-氰基取 代的化合物 101 (Scheme 11), 对这些化合物的体外抗肿 瘤活性研究表明, 具有 6-烷基氨基硫腙基取代的化合物
92,94 及化合物 102 具有很好的抑制肿瘤细胞生长增殖 活性, 它们对 Bel-7404 细胞的 IC50 值分别为 4.0, 7.2 和 $8.7 \mu \mathrm{mol} / \mathrm{L}$ ，优于阳性对照物顺铂.

另外, Kasal 等 ${ }^{[29]}$ 从乙酸-20-氧代-B-降孕-5-烯-3-酯 (20-oxo-7-nor-pregn-5-en-3 $\beta$-yl acetate) (103)出发，合成 了 B-失碳甾体化合物 104 113 (Scheme 12).

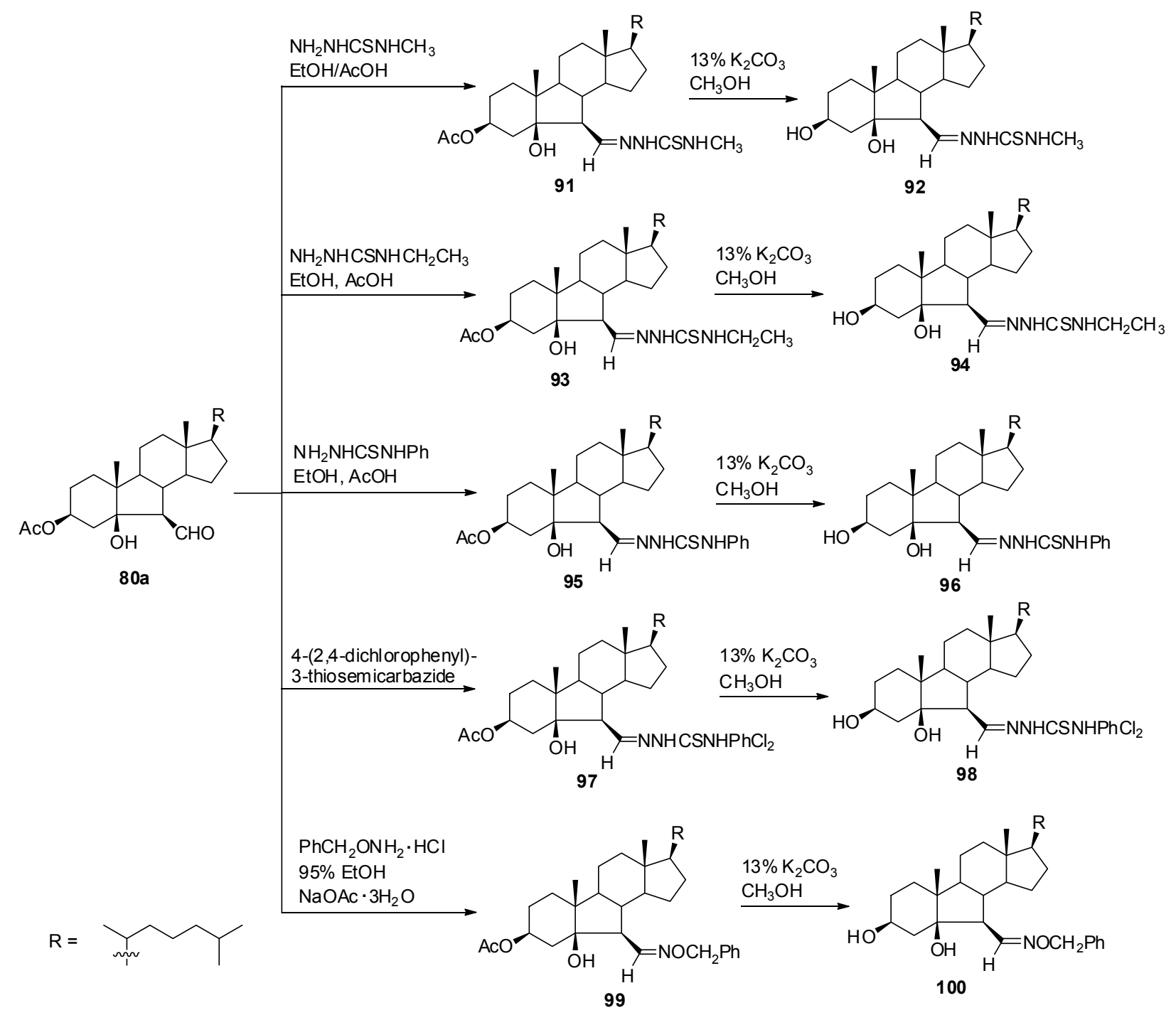

Scheme 10

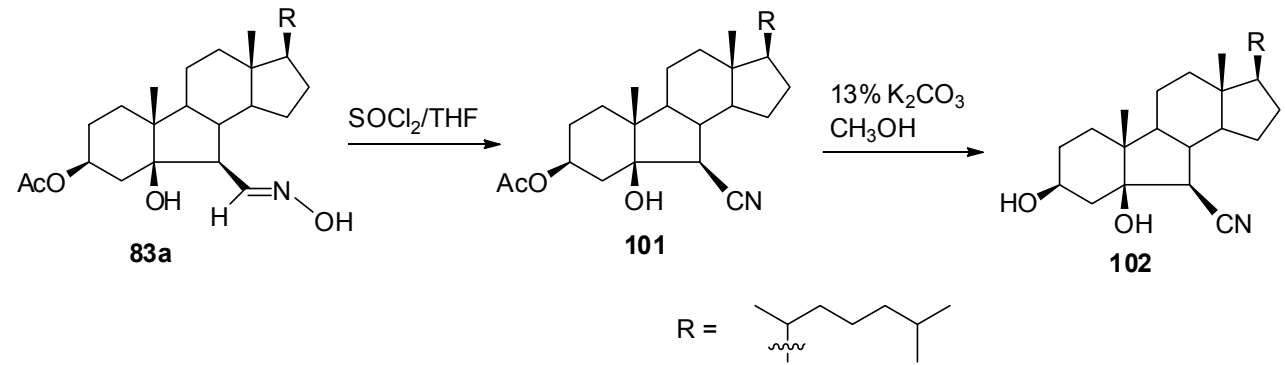

Scheme 11 


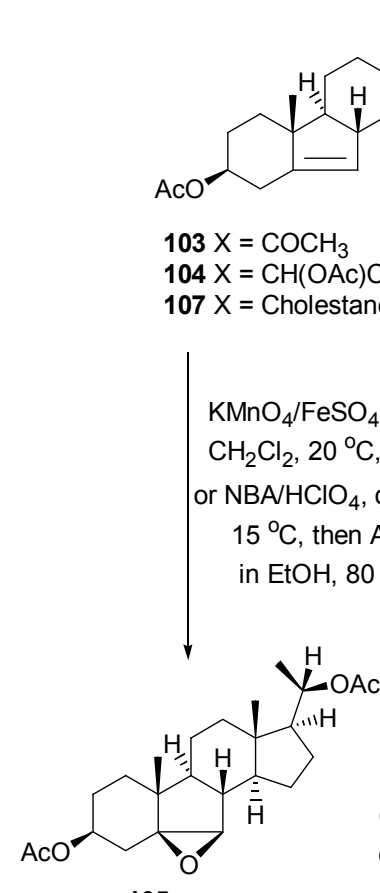

105 (i) $\mathrm{BF}_{3} \cdot \mathrm{Et}_{2} \mathrm{O}, \mathrm{THF}, 20^{\circ} \mathrm{C}, 20 \mathrm{~h}$

(ii) $\mathrm{NH}_{2} \mathrm{OH} \cdot \mathrm{HCl} / \mathrm{KHCO}_{3}$ boiling $\mathrm{MeOH}$ or EtOH, $0.25 \sim 5 \mathrm{~h}$ or O-(tert-butyl-dimethylsilyl)hydroxylamine toluene, $100^{\circ} \mathrm{C}, 4 \mathrm{~h}$, then $\mathrm{Bu}_{4} \mathrm{NF}$ in THF

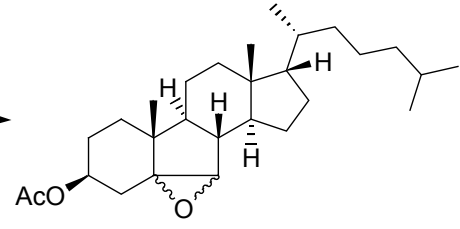

$1085 \alpha, 6 \alpha$-epoxide $1095 \beta, 6 \beta$-epoxide

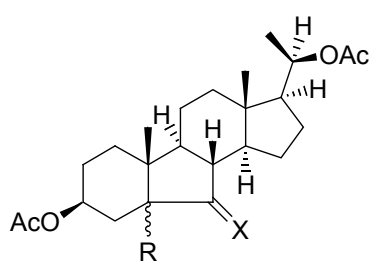

$106 \mathrm{R}=\alpha-\mathrm{H}, \mathrm{X}=\mathrm{O}$

$110 \mathrm{R}=\alpha-\mathrm{H}, \mathrm{X}=(Z)-\mathrm{NOH}$

$111 \mathrm{R}=\alpha-\mathrm{H}, \mathrm{X}=(E)-\mathrm{NOH}$

$112 \mathrm{R}=\alpha-\mathrm{H}, \mathrm{X}=\mathrm{NOH}$

$113 \mathrm{R}=\alpha-\mathrm{H}, \mathrm{X}=\operatorname{NPSi}\left(\mathrm{CH}_{3}\right)_{2} \mathrm{C}\left(\mathrm{CH}_{3}\right)_{3}$

Scheme 12

\section{C-失碳-D-增碳甾体化合物}

C-失碳-D-增碳(C-nor-D-homo)甾体化合物是甾体 骨架经过重排, C-环减少一个碳原子和 D-环增加一个碳 原子而得到的甾体化合物. 藜芦 Veratrum japonicum (Baker) Loes. f. 在中国的传统药物中是一种有名的毒物, Cong 等 ${ }^{[30]}$ 从这种植物的根茎中分离得到一种新物质 114 和 3 种已知物 115 117 (Chart 9), 这些化合物具有 C-失碳-D-增碳的 [6-6-5-6]的甾核结构, 并在 D-环上并 联了一个双环共享一个氮原子的全氢化芸杂环.
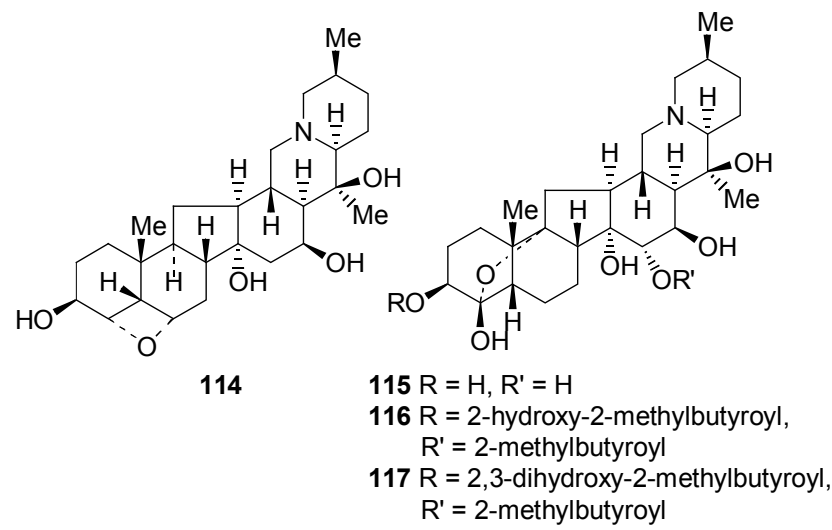

Chart 9

Veratrum nigrum L. 是百合科植物, 它是一种传统中
药, 可以用来治疗高血压, 中风, 羊癫疯等. Cong 等 ${ }^{[31]}$ 从 Veratrum nigrum L.中提取得到两种新的甾体生物碱 118, 119 (Chart 10), 并且测试了它们体外对人类神经胶 质瘤细胞株 SF188 的细胞毒性.

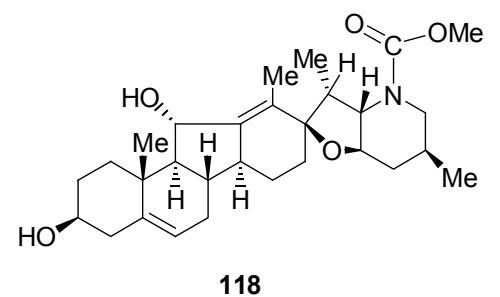

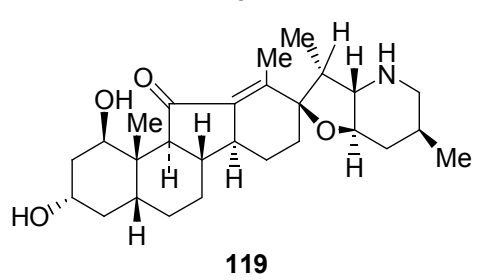

Chart 10

Zhang 等 ${ }^{[32]}$ 在分离贝母 Fritillaria hupehensis 的鳞茎 中分离得到化合物 120 125 (Chart 11), 同时还得到化 合物 118, 119. 测试这些化合物对人宫颈癌细胞 (HeLa) 和人肝癌细胞(HepG2)的细胞毒性, 化合物 $\mathbf{1 2 0}$ 和 $\mathbf{1 2 1}$ 显示了很好的细胞毒性, $\mathrm{IC}_{50}$ 值范围为 $2.52 \sim 0.23$ $\mu \mathrm{mol} / \mathrm{L}$, 与作为阳性对照的 5-fluorouracil 相似. 


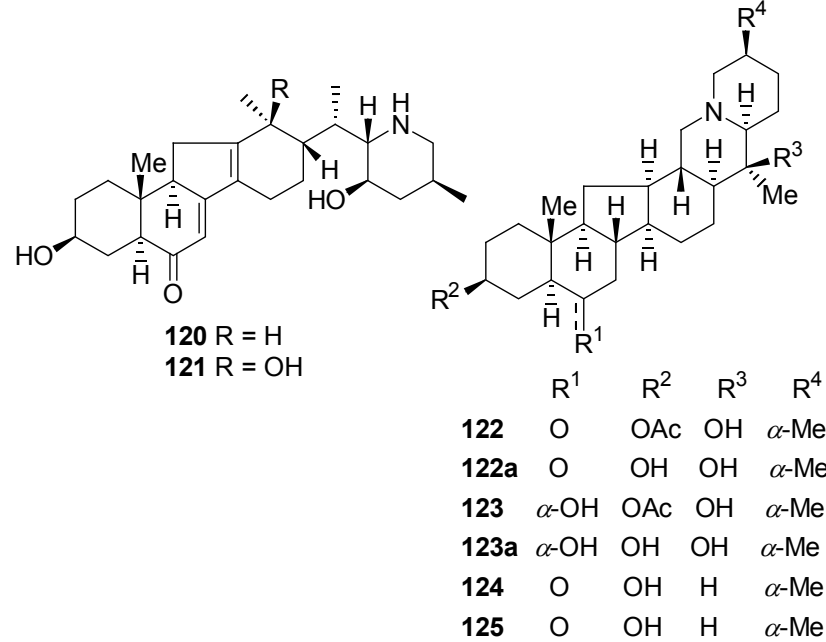

Chart 11

另外, Zhang 等 ${ }^{[33]}$ 从贝母 Fritillaria unibracteata 中 分离得到两种新的甾体葡萄糖苷生物碱 3-O- $\beta$ - D-glucopyranoside (126) 和 puqiedinone-3-O- $\beta$ - $D$-glucopyranoside (128)及 3 种已知化合物 127, 129, 130 (Chart 12). 化合物 128 对 PC12 细胞株显示出中等程度的神经毒活性.

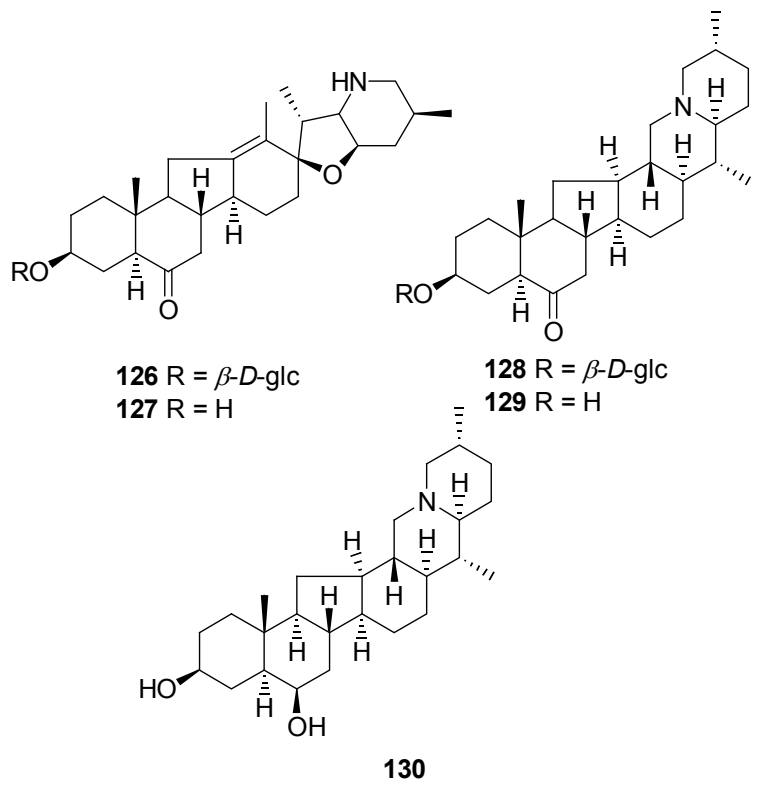

Chart 12

海绵中的黑皮海绵 Terpios hoshinota 会导致大量 珊瑚礁破坏, 通常称之为珊瑚黑病, 其严重影响了海洋 生态. 为了寻找 $T$. hoshinota 所产生的毒素, Uemura

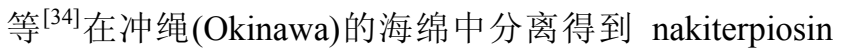
(131)和 nakiterpiosinone (132) (Chart 13), 他们表征了这 些复杂天然产物的结构, 结果表明化合物 132 是一种 C失碳-D-增碳甾体化合物. 对这些化合物进行生理活性 测试, 发现它们可以抑制小鼠白血病细胞 P388 的生长, 其 $\mathrm{IC}_{50}$ 值为 $15 \mathrm{nmol} / \mathrm{L}$.
在 C-失碳-D-增碳甾体化合物中, 最引人关注的是 化合物 cyclopamine (133) 和 veratramine (134) Chart $13^{[35]}$, 此两个化合物都属于 C-失碳-D-增碳甾体生物碱 类化合物, 它们存在于植物藜芦中. Binns 等发现给妊娠 的母羊喂加州㢣芦(Veratrum californicum), 出生的羔羊 是独眼, 而母羊本身不受影响，他们发现环巴胺 133 会 导致独眼脸畸形，而摄入黎芦胺 134 则导致腿畸形.

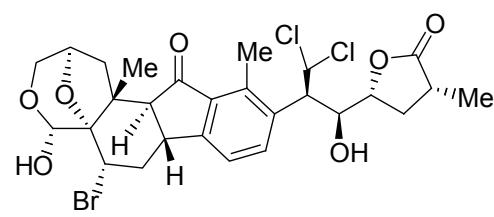

originally proposed nakiterpiosin (131)
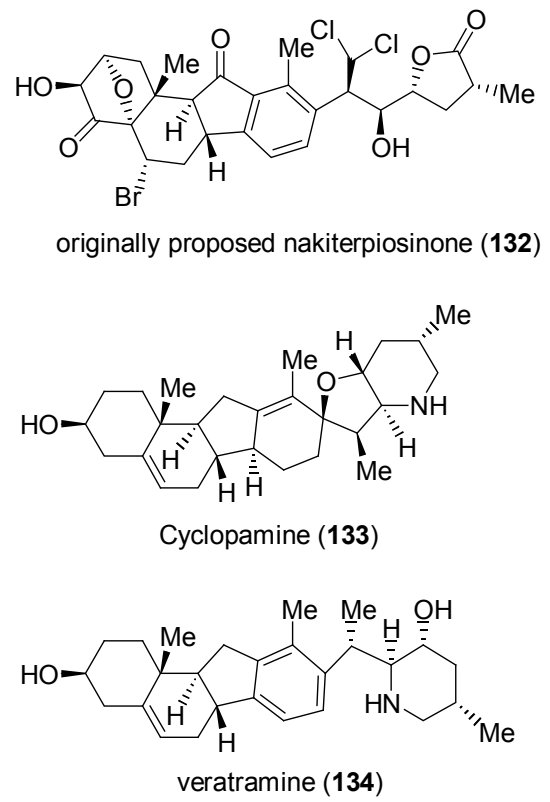

Chart 13

在 20 世纪 60 年代, cyclopamine (133), veratramine (134)和 jervine (11-oxo-134)的合成和结构鉴定是研究的 热点. 2008 年, 一种快速有效的分离 cyclopamine (133) 的方法被建立 ${ }^{[36]}$. 另外, 2009 年报道了一种更有效的化 合物 134 的合成方法 ${ }^{[37]}$.

Giannis 等 ${ }^{[38]}$ 用仿生合成的方法将普通甾体化合物 转变为 C-失碳-D-增碳-甾体化合物, 通过 Comins 试剂 和 4-( $N, N$ 二甲基氨基)吡啶的结合, 有效促进了甾体衍 生物的重排(Eq. 1), 得到具有 C-失碳-D-增碳-甾体产物, 但是在其它条件下重排不能进行。如化合物 135 以 $6: 1$ 的比例重排生成化合物 136 与 137.

Takamura 研究小组 ${ }^{[39]}$ 通过在 $160{ }^{\circ} \mathrm{C}$ 加热大环内酯 化合物 138, 使其发生分子内的 Diels-Alder 反应，得到 了较高产率的 139 和 140 非对映异构体混合物, 它们具 有 C-失碳-D-增碳甾体骨架的结构(Eq. 2). 


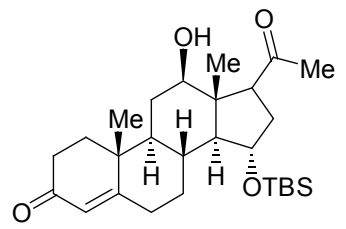

135

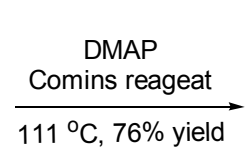

\section{西}$$
\text { 。 }
$$<smiles>CC(=O)C1C[C@H]([OH2+])[C@@H]2C(=C1C)C[C@H]1[C@@H]2CCC2=CC(=O)CC[C@@]21C</smiles>

136

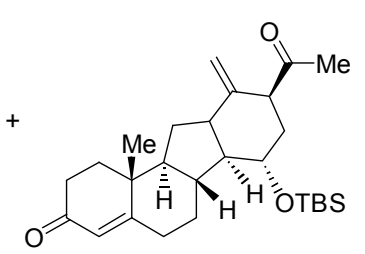

137

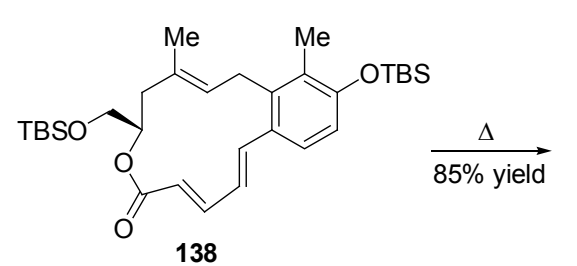

138

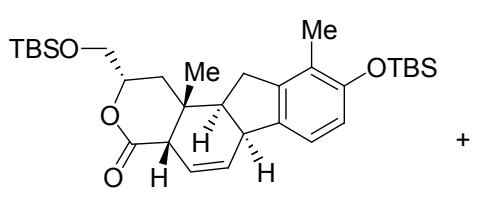

139

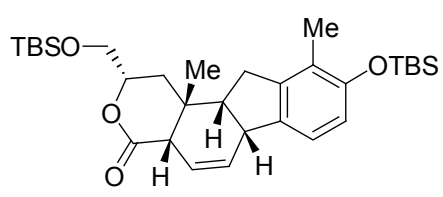

140
另外, Pattenden 等 ${ }^{[40]}$ 报道了通过串列式环化方法合 成此激素酮. 从底物 141 形成大环分子自由基, 然后经 过双跨环成环反应, 并从三丁基氢化锡中脱氢得到化合 物 142 和 143 及大环分子 144 , 化合物 142 和 143 具有 C-失碳-D-增碳甾体骨架结构(Eq. 3).<smiles>C=CC1CC1c1ccccc1CC=C(C)CCC(=O)P</smiles>

141

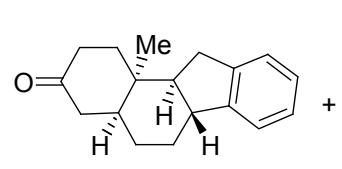

143

$n(\mathbf{1 4 2}): n(\mathbf{1 4 3 )}: n(\mathbf{1 4 4 )}=1: 1: 2$

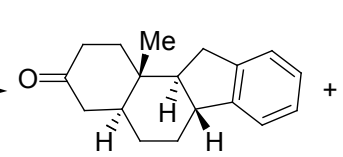

142

\section{4 启示与展望}

综上所述，具有特殊甾核结构的甾体化合物中，大 多都表现出良好的抗肿瘤、抗菌、抗炎等生物活性. 通 过对具有生物活性的天然甾体化合物进行结构改造, 改 变甾核结构, 引入具有较高生理活性的官能团, 进一步 对其进行活性篮选, 有利于抗肿瘤新药的研究开发 ${ }^{[41 \sim 43]}$. 然而, 文献中对此类化合物的构效关系方面的研究报道 不多. 目前, 对于 B-环失碳甾体化合物的合成及生物活 性研究已逐渐引起化学及药物学家的重视, 我们发现, 当甾体 17-支链为长链烷基支链结构, B-环失碳环中 6位取代基为着甲基、肜基、氰基、氨基硫腙基或烷基取 代的氨基硫腙基结构时, 化合物都具有比较好的抑制肿 瘤细胞生长增殖活性. 而当 17 -支链为极性官能团支链 结构时, 化合物抑制肿瘤细胞生长增殖活性明显降低甚 至无活性. 因此, 将甾体支链结构固定为烷基支链结构
(如胆甾烷或谷甾烷支链结构), 然后将甾核改造为 B-环 失碳的 [6-5-6-5]-甾核, 在甾核的不同位置进一步引入活 性官能团, 探讨其抗肿瘤活性机理, 将有利于篮选出具 有更高生物活性的甾体类化合物.

另外，除了通过采取缩碳或增碳的方式去改变甾核 的结构, 以改变甾体母体化合物的生物功能外. 也可以 通过利用杂原子来取代甾核中的碳原子 ${ }^{[44 ~ 46]}$ 或在甾核 中引入不同的杂原子以形成含杂原子的扩环甾核 ${ }^{[47,48]}$, 去进一步增强甾体化合物在某个方面的生物活性. 我们 分别对某些 A-homo ${ }^{[49,50]}, \mathrm{B}-$ homo o $^{[51]}, \mathrm{C}-$ homo $^{[52]}$ 及 D-homo ${ }^{[53]}$ 类型的甾体扩环内酰胺化合物及 B-homo ${ }^{[54]}$ 类型甾体扩环内酯化合物进行了合成及抗肿瘤活性研 究, 结果表明具有胆甾烷支链结构的化合物均具有较好 的抑制肿瘤细胞生长增殖活性，对肿瘤细胞的抑制作用 主要通过诱导细胞调亡的方式, 某些A-nomo 及 B-homo 内酰胺化合物在动物模型中对移植肿瘤也表现出一定 的抑制作用. 由此可见，对于具有特殊甾核结构甾体化 合物的研究来说, 保持胆甾烷类型的 17-支链结构, 然 后对甾核结构进行不同的改造或修饰，可期望获得具有 更好抗肿瘤活性的此类化合物.

\section{References}

[1] Zhang, D. Y.; Xu, M. X.; Zheng, H. West China J. Pharm. Sci. 2000, 15, 189 (in Chinese).

(张大永, 徐鸣夏, 郑虎, 华西药学杂志, 2000, 15, 189.)

[2] Zhang, L.; Weng, S. M.; Weng, X. H. J. Fujian Med. Univ. 2008, 42, 208 (in Chinese).

(张立, 翁绳美, 翁秀华, 福建医科大学学报, 2008, 42, 208.)

[3] Liu, J.; Zheng, W.; Chen, H. L.; Chen, J. X. Reprod. Contracep. 2005, 25, 272 (in Chinese).

(刘静, 郑伟, 陈海林, 陈建兴, 生殖与避孕, 2005, 25, 272.)

[4] Zhang, P. Y.; Chen, H. L.; Chen, J. X. Reprod. Contracep. 2008, 28, 588 (in Chinese).

(张培翼, 陈海林, 陈建兴, 生殖与避孕, 2008, 28, 588.) 
[5] Rhonda, O.; Maria, F. Drugs 2004, 64, 725.

[6] Li, Z. T.; Liao, Q. J. Chin. New Drugs J. 2004, 13, 720 (in Chinese). (李宗桃, 廖清江, 中国新药杂志, 2004, 13, 720.)

[7] Zhao, Y. P.; Li, W.; Lü, L.; Qu, J.; Chang, Y. Z.; Ding, C.; Li, Z. T. Chin. J. Med. Chem. 2010, 20, 501 (in Chinese). (赵滟萍, 黎万, 吕露, 屈洁, 常永志, 丁聪, 李宗桃, 中国药物 化学杂志, 2010, 20, 501.)

[8] Grosjean, E.; Love, G. D.; Stalvies, C.; Fike, D. A.; Summons, R. E. Org. Geochem. 2009, 40, 87.

[9] Gaines, S. M.; Eglinton, G.; Rullkötter, J. Echoes of Live, What Fossil Molecules Reveal About Earth History, Oxford University Press, New York, 2008.

[10] Norden, S.; Bender, M.; Rullkötter, J.; Christoffers, J. Eur. J. Org. Chem. 2011, 4543.

[11] Nowrouzi, F.; Janetzko, J.; Batey, R. A. Org. Lett. 2010, 12, 5490.

[12] Qiu, Y.; Deng, Z. W.; Xu, M. J.; Li, Q. S.; Lin, W. H. Steroids 2008, 73, 1500 .

[13] Pointinger, S.; Promdang, S.; Vajrodaya, S.; Pannell, C. M.; Hofer, O.; Mereiter, K.; Greger, H. Phytochemistry 2008, 69, 2696.

[14] Miyamoto, T.; Kodama, K.; Aramaki, Y.; Higuchi, R.; Van Soest, R. W. M. Tetrahedron Lett. 2001, 42, 6349.

[15] Liu, B.; Zhou, W. S. Tetrahedron Lett. 2002, 43, 4187.

[16] Liu B.; Zhou, W. S. Tetrahedron 2003, 59, 3379.

[17] Wentworth, J. P.; Nieva, J.; Takeuchi, C.; Galve, R.; Wentworth, A. D.; Dilley, R. B.; DeLaria, G. A. Saven, A.; Babior, B. M.; Janda, K. D.; Eschenmoser, A.; Lerner, R. A. Science 2003, 302, 1053.

[18] Paul, W.; San, D. WO 2007022089, 2007 [Chem. Abstr. 2007, 146, 244369].

[19] Brinkhorst, J.; Nara, S. J.; Pratt, D. A. J. Am. Chem. Soc. 2008, 130, 12224.

[20] Suñol, C.; García, D. A.; Bujons, J.; Kristofiková, Z.; Matyás, L.; Babot, Z.; Kasal, A. J. Med. Chem. 2006, 49, 3225.

[21] Wei, X.; Rodríguez, A. D.; Wang Y.; Franzblau, S. G. Bioorg. Med. Chem. Lett. 2008, 18, 5448.

[22] Shukla, Y. J.; Pawar, R. S.; Ding, Y.; Li, X. C.; Ferreira, D.; Khan, I. A. Phytochemistry 2009, 70, 675.

[23] Cygan, N. K.; Scheinost, J. C.; Butters, T. D.; Wentworth, P. Biochemistry 2011, 50, 2092.

[24] Gan, C. F.; Fan, L. H.; Cui, J. G.; Huang, Y. M.; Jiao, Y. X.; Wei, W. Steroids 2012, 77, 1061.

[25] Gan, C. F.; Fan, L. H.; Huang, Y. M.; Liu, Z. P.; Cui, J. G. Med. Chem. 2013, 9, 546

[26] Gan, C. F.; Li, W. Y.; Lin, Q. F.; Huang, Y. M.; Cui, J. G. Chem. Reag. 2013, 35, 218 (in Chinese).

(甘春芳, 李伟玉, 林啟福, 黄燕敏, 崔建国, 化学试剂, 2013, 35, 218.)

[27] Gan, C. F.; Liu, Z. P.; Wei, W. X.; Huang, Y. M.; Cui, J. G. Chem. Res. Appl. 2013, 25, 647 (in Chinese). (甘春芳, 刘志平, 韦万兴, 黄燕敏, 崔建国, 化学研究与应用, 2013, 25, 647.)

[28] Gan, C. F.; Lin, Q. F.; Cui, J. G.; Feng, J. D.; Guo, J. N.; Liao, H. Y.; Huang, Y. M. Steroids 2014, 79, 37

[29] Kasal, A.; Krištofíková, Z.; Buděšíský, M. Tetrahedron 2007, 63, 11355

[30] Cong, Y.; Guo, L.; Yang, J. Y.; Li, L.; Zhou, Y. B.; Chen, J.; Wang, J. H. Planta Med. 2007, 73, 1588.

[31] Cong, Y.; Jia, W.; Chen, J.; Song, S.; Wang, J. H.; Yang, Y. H.
Helv. Chim. Acta 2007, 90, 1038.

[32] Zhang, Y. H.; Yang, X. L.; Zhang, P.; Zhou, X. F.; Ruan, H. L.; Pi, H. F.; Wu, J. Z.; Sun, H. D. Chem. Biodiversity 2008, 5, 259

[33] Zhang, Q. J.; Zheng, Z. F.; Yu, D. J. Asian Nat. Prod. Res. 2011, 13, 1098.

[34] Tang, S. L.; Hong, M. J.; Liao, M. H.; Jane, W. N.; Chiang, P. W.; Chen, C. B.; Chen, C. A. Environ. Microbiol. 2011, 13, 1179.

[35] (a) Teruya, T.; Nakagawa, S.; Koyama, T.; Suenaga, K.; Kita, M.; Uemura, D. Tetrahedron Lett. 2003, 44, 5171.

(b) Teruya, T.; Nakagawa, S.; Koyama, T.; Arimoto, H.; Kita, M.; Uemura, D. Tetrahedron 2004, 60, 6989.

[36] Oatis, J. E. Jr.; Brunsfeld, P.; Rushing, J. W.; Moeller, P. D.; Bearden, D. W.; Gallien, T. M. Chem. Cent. J. 2008, $2,12$.

[37] Giannis, A.; Heretsch, P.; Sarli, V.; Stößel, A. Angew Chem., Int. Ed. 2009, 48, 7911.

[38] Heretsch, P.; Rabe, S.; Giannis, A. J. Am. Chem. Soc. 2010, 132, 9968.

[39] (a) Ito, T.; Ito, M.; Arimoto, H.; Takamura, H.; Uemura, D. Tetrahedron 2007, 48, 5465.

(b) Takamura, H.; Yamagami, Y.; Ito, T.; Arimoto, H.; Kadota, I.; Uemura, D. Heterocycles 2009, 77, 351.

[40] (a) Pattenden, G.; Wiedenau, P. Tetrahedron Lett. 1997, 38, 3647.

(b) Pattenden, G.; Gonzalez, M. A.; McCulloch, S.; Walter, A.; Woodhead, S. J. Proc. Natl. Acad. Sci. U. S. A. 2004, 101, 12024.

[41] Chen, S. J.; Cui, J. G.; Li, Y.; Fan, L. H. Chin. J. Org. Chem. 2011, 31,187 (in Chinese). (陈思静，崔建国，李芗，范良华，有机化学, 2011, 31, 187.)

[42] Zhang, X. J.; Cui, J. G.; Li, Y.; Chen, S. J. Chin. J. Org. Chem. 2010, 30, 655 (in Chinese) (张晓佳, 崔建国, 李芗, 陈思静, 有机化学, 2010, 30, 655.)

[43] Lin, Q. F.; Cui, J. G.; Gan, C. F.; Liu, L.; Yao, Q. C.; Huang, Y. M. Chin. J. Org. Chem. 2012, 32, 2214 (in Chinese). (林啟福，崔建国，甘春芳，刘亮，姚秋翠，黄燕敏，有机化学， 2012, 32, 2214.)

[44] Ibrahim-Ouali, M.; Rocheblave, L. Steroids 2008, 73, 375.

[45] Ibrahim-Ouali, M. Steroids 2007, 72, 475.

[46] Ibrahim-Ouali, M.; Santelli, M. Steroids 2006, 71, 1025.

[47] Huang, Y. M.; Cui, J. G.; Gan, C. F.; Yao, Q. C.; Jia, L. Y. Prog. Chem. 2012, 24, 61 (in Chinese).

(黄燕敏, 崔建国, 甘春芳, 姚秋翠, 贾琳怡, 化学进展, 2012, 24, 61.)

[48] Gan, C. F.; Cui, J. G.; Huang, Y. M.; Wei, W. X.; Fan, L. H. Chemistry 2011, 74, 926 (in Chinese).

(甘春芳，崔建国，黄燕敏，韦万兴，范良华，化学通报，2011, 74, 926).

[49] Huang, Y. M.; Chen, S. J.; Cui, J. G.; Gan, C. F.; Liu, Z. P.; Wei, Y. L.; Song, H. C. Steroids 2011, 76, 690.

[50] Huang, Y. M.; Cui, J. G.; Chen, S. J.; Gan, C. F.; Zhou, A. M. Steroids 2011, 76, 1346.

[51] Huang, Y. M.; Cui, J. G.; Chen, S. J.; Lin, Q. F.; Song, H. C.; Gan, C. F.; Su, B.; Zhou, A. M. Mar. Drugs 2014, 12, 1715.

[52] Huang, Y. M.; Cui, J. G.; Chen, S. J.; Gan, C. F.; Yao, Q. C.; Lin, Q. F. Bioorg. Med. Chem. Lett. 2013, 23, 2265.

[53] Huang, Y. M.; Cui, J. G.; Zhong, Z. G.; Gan, C. F.; Zhang, W. Y.; Song, H. C. Bioorg. Med. Chem. Lett. 2011, 21, 3641.

[54] Gan, C. F.; Cui, J. G.; Huang, Y. M.; Jia, L. Y.; Wei, W. X. Steroids 2012, 77, 255. 